East African Medical Journal Vol. 80 No. 1 January 2003

INDUCTION OF LABOUR USING LOW AND HIGH DOSE REGIMENS OF PROSTAGLANDIN E2 VAGINAL TABLETS

A.M. Bahar, FRCOG, Associate Professor, E.I. Archibong FRCOG, Consultant Obstetrician and Gynaecologist, Abha Maternity Hospital, Abha, Saudi Arabia, Z.M.S Zaki, FRCOG, Associate Professor and Consultant, Department of Obstetrics and Gynaecology, College of Medicine and Medical Sciences, King Khalid University and Abha Maternity Hospital, Abha, Saudi Arabia and A.A. Mahfouz, Professor, Department of Community Medicine, College of Medicine and Medical Sciences, King Khalid University, Abha, Saudi Arabia

Request for reprints to: Dr. A.M. Bahar, College of Medicine, King Khalid University, P.O.Box 641, Abha, Saudi Arabia

\title{
INDUCTION OF LABOUR USING LOW AND HIGH DOSE REGIMENS OF PROSTAGLANDIN E2 VAGINAL TABLETS
}

\author{
A.M. BAHAR, E.I. ARCHIBONG, Z.M.S ZAKI and A.A. MAHFOUZ
}

\begin{abstract}
Objective: To compare the efficacy of low dose prostaglandin $E_{2}$ vaginal tablets with that of high dose in induction of labour.

Design: A retrospective study.

Setting: Abha Maternity Hospital, a teaching hospital in the southern region of Saudia Arabia.

Patients: The outcome of induction of labour in 73 women induced with $1.5 \mathrm{mg}$ prostaglandin $E_{2}$ vaginal tablets was compared with those in 168 women induced with $3 \mathrm{mg}$ vaginal tablets.

Results: There were no statistical significant differences in the success rate, inductiondelivery interval, caesarean section rate and Apgar scores between the two groups. There were two incidences of uterine hyperstimulation and two of uterine rupture in the high dose group. These were not statistically significant. Logistic regression analysis showed that the success rate of induction of labour was dependent only on maternal age $(\mathrm{P}=\mathbf{0 . 0 0 2 5})$ and Bishop score $(\mathrm{P}=\mathbf{0 . 0 4 0 3})$ and not on parity, gestational age, birthweight or dose of prostaglandin.

Conclusion: The low dose regimen of $1.5 \mathrm{mg}$ is as efficacious as the high dose regimen of 3mg. prostaglandin $E_{2}$ vaginal tablets in inducing labour. This implies that using the low dose regimen reduces the cost of induction of labour effectively. Larger prospective randomized studies are needed to confirm this finding.
\end{abstract}

\section{INTRODUCTION}

The rates and indications for induction of labour have increased recently resulting in increasing number of women with higher parity and older age being induced(1). For the last two decades prostaglandins had become popular as inducing agents and are used worldwide. The developing countries are no exception, and with their limited resources induction of labour may impose financial burden on health authorities. The proportion of women with high parity is high in these communities and the experience in induction of labour with prostaglandins is also limited. About $30 \%$ of pregnant women attending this institution are grand multiparous (parity $>5$ ). The initial report about safety of induction of labour with pharmacy prepared prostaglandin $\mathrm{E}_{2}$ vaginal pessaries in grand multiparous women in the Middle East came from Kuwait(2). Prostaglandin tablets were not available then. Prostaglandin E2 (dinoprostone) vaginal tablets are now widely available, and have the advantages of availability, stability and ease of administration. Induction of labour with traditional doses of prostaglandin $\mathrm{E}_{2}$ had been reported to lead to uterine hyperstimulation leading to fetal asphyxia(3) and sometimes uterine rupture(4) particularly in the multigravid woman or women with previous caesarean sections(5-7). In an effort to find out whether a lower dose regimen of prostaglandin $\mathrm{E}_{2}$ vaginal tablets is as efficacious as a higher dose regimen, and with less complications, especially in our environment of high parity women, we reviewed retrospectively all prostaglandin inductions carried out in Abha maternity hospital, Saudi Arabia, in an 18 month period where patients were induced with either low or high dose prostaglandin E2 vaginal tablets.

\section{MATERIALS AND METHODS}

All the records of patients who underwent induction of labour with prostaglandin $\mathrm{E}_{2}$ vaginal tablets during the period between 15 November 1996 and 15 May 1998 were reviewed. There were 297 patients. Patients selected for the study fulfilled the following criteria: gestational age 37-42 weeks, singleton pregnancies with cephalic presentation, intact membranes, a living fetus and no contraindication to vaginal delivery or prostaglandin administration. Patients with a history of one previous caesarean section were also included. Patients who were excluded from the study were: 21 patients with gestational age less than 37 weeks, 25 patients with 
premature rupture of the membranes, eight patients in whom the protocol was not strictly followed and two patients with breech presentation. In all, 241 patients fulfilled the criteria for inclusion in the study; eight of these had one previous caesarean section. Patients were either induced with a dose of 3mg prostaglandin E2 (PGE2) [Dinoprostone, UpJohn, $\mathrm{UK}$ ] vaginal tablets or $1.5 \mathrm{mg}$ (half a tablet). Our protocol allowed either dose to be used, and the decision on which dose to use was left to the discretion of the attending consultant. In general consultants were biased towards giving high parity women the lower dose. One hundred and sixty eight women received $3 \mathrm{mg}$ dose regimen (high dose group) and 73 patients received $1.5 \mathrm{mg}$ dose regimen (low dose group). The tablets were inserted high in the posterior vaginal fornix by a senior registrar or a consultant after assessing the cervical status using the modified Bishop score. After insertion of the tablet the patient remained in bed for one hour and the fetus was monitored by external cardiotocography during that period. If the patient did not develop contractions or the attending specialist considered the contractions weak six hours after the first insertion the patient received another insertion of prostaglandin of the same dose after cervical assessment. If the same situation prevailed six hours after the second insertion, a third dose was inserted. No further insertion was made thereafter in the first 24 hours. Oxytocin was not administered as per protocol. Amniotomy was not used to accelerate labour. It was only carried out in women with established labour having abnormality in the cardiotocographic trace to check for meconium stained liquor. Patients who did not deliver within 24 hours after the first insertion of prostaglandin were managed next day according to the discretion of the attending consultant. Demographic data about the patients as well as the course of labour, delivery and complications were collected from the patients files. Induction was considered successful if the patient achieved vaginal delivery within 24 hours of first insertion of prostaglandin. The primary outcome of interest was the success of induction. Secondary outcomes of interest were induction-delivery interval, maternal complications and newborn Apgar score at delivery.

Statistical analysis was carried out using SPSS Release 9. For continuous variables the Student's t-test was used and for categorical variables the $\chi 2$ test or Fisher's exact test were used when appropriate. To control for the effects of potential independent confounding variables binary logistic regression analysis was used with success rate as the dependent variable(8). The independent confounding variables analysed were age ( $\leq 30$ years Vs $>30$ years), parity $(\leq 5 \mathrm{Vs}$ $>5$ ), gestational age ( $\leq 40$ weeks $V s>40$ weeks), Bishop scores $(\leq 5 \mathrm{Vs}>5)$, dose of prostaglandin $(1.5 \mathrm{mg} \mathrm{Vs} 3 \mathrm{mg})$ and birth weight $(\leq 3 \mathrm{~kg} \mathrm{Vs}>3 \mathrm{~kg}$ ). The level of significance was set at $\mathrm{p}<0.05$.

\section{RESULTS}

Comparison of maternal characteristics between the two groups is shown in Table 1. Women who received the low dose regimen had significantly higher mean age and parity and received less total prostaglandin dose. The indications for induction of labour did not differ between the groups (Table 2).

Table 1

Maternal characteristics

\begin{tabular}{|c|c|c|c|}
\hline Characteristic & $\begin{array}{c}1.5 \mathrm{mg}(\mathrm{n}=73) \\
\text { mean } \pm \mathrm{SD}\end{array}$ & $\begin{array}{c}3 \mathrm{mg}(\mathrm{n}=168) \\
\text { mean } \pm \mathrm{SD}\end{array}$ & P-value \\
\hline Age (years) & $33.47 \pm 6.41$ & $28.05 \pm 5.49$ & 0.0001 \\
\hline Parity & $6.79 \pm 2.83$ & $2.79 \pm 2.65$ & 0.0001 \\
\hline Gestational age (weeks) & $40.11 \pm 1.47$ & $40.21 \pm 1.55$ & NS \\
\hline Bishop score & $4.81 \pm 1.28$ & $4.74 \pm 1.47$ & NS \\
\hline PGE2 total dose (mg) & $2.94 \pm 1.23$ & $5.7 \pm 2.38$ & 0.0001 \\
\hline
\end{tabular}

$\mathrm{NS}=$ not significant $\mathrm{SD}=$ Standard deviation $\mathrm{PGE} 2=$ Prostaglandin $\mathrm{E}_{2}$

Table 2

Indications for induction of labour

\begin{tabular}{|c|c|c|c|}
\hline Indication & $\begin{array}{c}1.5 \mathrm{mg} \text { group } \\
(\mathrm{n}=73) \\
\text { No. }(\%)\end{array}$ & $\begin{array}{c}3 \text { mg group } \\
(n=168) \\
\text { No. }(\%)\end{array}$ & $\begin{array}{c}\text { Total }(\mathrm{n}=141) \\
\text { No. }(\%)\end{array}$ \\
\hline Prolonged gestation & $40(54.8)$ & $83(49.4)$ & $123(51.0)$ \\
\hline Diabetes mellitus & $11(15.1)$ & $24(14.3)$ & $35(14.5)$ \\
\hline Hypertensive diseases & $9(12.3)$ & $20(11.9)$ & $29(12.0)$ \\
\hline Intrauterine growth retardation & $7(9.6)$ & $11(6.5)$ & $18(7.5)$ \\
\hline Others* & $6(8.2)$ & $30(17.9)$ & $36(14.9)$ \\
\hline
\end{tabular}

*poor obstetric history, non-assuring biophysical profile, Rh isoimmunization 
Maternal and foetal outcomes as well as complications of induction of labour are shown in Table 3. The only difference between groups was in the newborn birthweight, being significantly higher in the low dose group. Eighty nine per cent of the low dose regimen group and $82 \%$ of the high dose regimen group achieved vaginal delivery within 24 hours of induction, while the rates of caesarean sections were $8.2 \%$ and $11.3 \%$ respectively. The mean inductiondelivery interval for women who achieved vaginal delivery did not differ between the groups. The mean Apgar score for the newborns of women who achieved vaginal delivery did not differ also. No babies in either group had an Apgar score less than 7 in 5 minutes. There was no perinatal mortality. There were two patients who sustained posterior uterine rupture in the first stage of labour. One was para 5 and the other was para 10. Both were from the high dose group and both received two doses. None of them had a previous caesarean section. One of them, the para 5, had a history of uterine curettage for an incomplete miscarriage. There were two incidences of uterine hyperstimulation both from the high dose group and three incidences of atonic primary postpartum haemorrhage, one being from the low dose group.

As the two groups differed in maternal age, parity, newborn birthweight and prostaglandin dose, a binary logistic regression analysis, with the success rate as the dependent variable, was carried out to control for the effect of these as well as other confounding factors which might have affected the success of induction of labour. This has shown the success rate to be dependent only on maternal age and initial Bishop score and not on parity, gestational age, birthweight or the dose of prostaglandin. The success rate was significantly decreased in older women $(>30)$ compared with the younger ones $(\mathrm{OR}=4$. $\mathrm{P}=0.0025)$ and also in women with unripe cervices (Bishop score $\leq 5)$ compared with those with ripe cervices ( $\mathrm{OR}=0.36$ $\mathrm{P}=0.0403$ ) (Table 4).

Table 3

Maternal and foetal outcomes

\begin{tabular}{lccc}
\hline Outcome & $\begin{array}{c}1.5 \mathrm{mg} \text { group } \\
(\mathrm{n}=73)\end{array}$ & $\begin{array}{c}3 \mathrm{mg} \text { group } \\
(\mathrm{n}=168)\end{array}$ & P-value \\
\hline Spontaneous vaginal delivery $\mathrm{n}(\%)$ & $65(89)$ & $138(82.1)$ & $\mathrm{NS}$ \\
Caesarean section $\mathrm{n}(\%)$ & $6(8.2)$ & $19(11.3)$ & $\mathrm{NS}$ \\
Uterine hyperstimulation n(\%) & 0 & $2(1.2)$ & $\mathrm{NS}$ \\
Abruptio placentae n(\%) & $1(1.4)$ & 0 & $\mathrm{NS}$ \\
Postpartum haemorrhage n(\%) & $1(1.4)$ & $2(1.2)$ & $\mathrm{NS}$ \\
Ruptured uterus $\mathrm{n}(\%)$ & 0 & $2(1.2)$ & $\mathrm{NS}$ \\
Induction-delivery interval (hours) & $13.40 \pm 10.8$ & $\mathrm{NS}$ \\
(mean \pm SD) & $3197.30 \pm 496$ & $13.14 \pm 6.7$ & 0.006 \\
Birthweight (g) (mean \pm SD) & $6.85 \pm 0.59$ & $3001.50 \pm 512$ & $\mathrm{NS}$ \\
Apgar score in 1 minute (mean \pm SD) & $8.95 \pm 0.37$ & $6.79 \pm 0.66$ & $\mathrm{NS}$ \\
Apgar score in 5 minutes (mean \pm SD) & $8.89 \pm 0.36$ & \\
\hline
\end{tabular}

$\mathrm{NS}=$ Not significant

$\mathrm{SD}=$ Standard deviation

Table 4

P-value, Odds ratio and $95 \%$ confidence interval for independent confounding factors included in logistic regression model to examine their effects on success of induction of labour

\begin{tabular}{lccc}
\hline Variable & P-value & Odds ratio & $\begin{array}{c}95 \% \text { Confidence } \\
\text { interval }\end{array}$ \\
\hline Maternal age & & & $0.09-0.60$ \\
Parity & 0.0025 & 0.24 & $0.19-1.67$ \\
Gestational age & NS & 0.57 & $0.98-4.65$ \\
Bishop score & NS & 2.14 & $0.14-0.95$ \\
Dose of PGE2 & 0.0403 & 0.36 & $0.87-6.53$ \\
Newborn birthweight & NS & 2.38 & $0.70-3.43$ \\
\hline
\end{tabular}

NS $=$ Not significant $\quad$ PGE2 $=$ Prostaglandin E2 
In a univariate analysis when women whose ages were 35 years or more $(n=65)$ were compared with those whose ages were less than 35 years $(n=176)$, it was found that the elder women had significantly less success rate [49(75.4\%) vs $154(87.5 \%) \mathrm{p}=0.022]$. When women with parity of 5 or less $(n=164)$ were compared with women whose parity was more than $5(\mathrm{n}=77)$, no difference was found in success rate $(84.1 \%$ Vs $84.4 \%)$. No difference in success rate was found when women with a parity of 3 or less $(n=123)$ were compared with women whose parity was more than $3 \quad(n=118)$ [102(82.9\%) vs $101(85.6 \%)]$. When primigravidae women were removed from the study and all the analyses shown above were repeated, similar results were obtained.

Of the 38 women who had failed induction, 18 did not show a satisfactory response to prostaglandin. When these poor responders were compared with those who had good response to prostaglandin, it was found that the poor responders had significantly higher number of primigravidae, women aged 35 years and above and women with lower Bishop score (Table 5). Fourteen (77.5\%) of them belonged to the high dose group and four $(22.5 \%)$ to the low dose group. This difference was not significant. Seven of the nine women whose age was 35 years or older were multigravidae with a mean parity of $8.43 \pm 4.2$ (range $1-13$ ) and mean age of $37.71 \pm 2.3$ (range 35-40).

Table 5

Comparison between patients who failed to initiate or develop adequate uterine contractions with those who developed adequate uterine contractions

\begin{tabular}{|c|c|c|c|}
\hline Variable & $\begin{array}{c}\text { Non- } \\
\text { responders } \\
(\mathrm{n}=18) \\
\text { No. }(\%)\end{array}$ & $\begin{array}{c}\text { Responders } \\
(\mathrm{n}=223) \\
\text { No. }(\%)\end{array}$ & P-value \\
\hline Primigravidae & $8(44.4)$ & $38(17.0)$ & 0.009 \\
\hline Maternal age $\geq 35$ years & s $9(50.0)$ & $56(25.1)$ & 0.029 \\
\hline Bishop score $<6$ & $18(100)$ & $152(68.2)$ & 0.004 \\
\hline $\begin{array}{l}\text { Total PGE2 dose } \\
\text { more than } 4.5 \mathrm{mg} \\
\text { Gestational age }\end{array}$ & $14(77.8)$ & $94(42.2)$ & 0.003 \\
\hline$>40$ weeks & $12(66.0)$ & $108(48.4)$ & NS \\
\hline
\end{tabular}

$\mathrm{NS}=$ Not significant PGE2 $=$ Prostaglandin E2

Seven of the eight women with previous caesarean sections belonged to the high dose group. All delivered vaginally except two who were delivered by caesarean section, one for failure to respond adequately to prostaglandin after the third dose and the other for severe lower abdominal tenderness after receiving the first dose. The uterus of this patient was found intact during caesarean section. The woman who belonged to the low dose group delivered vaginally. The mean parity of these patients was $3.63 \pm 2$ (range 1-7) and the mean age was $29.25 \pm 4.8$ (range $23-36$ ). None of the women who delivered in the first 24 hours needed operative vaginal delivery.

\section{DISCUSSION}

The study has shown no difference in the incidence of successful induction, caesarean section, inductiondelivery interval and foetal outcome between the 1.5 $\mathrm{mg}$ group and $3 \mathrm{mg}$ group. The primary aim of the study was to find out whether the low dose regimen is as efficient as the high dose regimen. It may be argued that the low dose group achieved comparable success to the high dose group because of their higher parity making them more sensitive to prostaglandin. However in the logistic regression model, after controlling for the effects of confounding factors, only maternal age and initial Bishop scores turned out to be significantly affecting the success rate. The other confounding factors including parity and prostaglandin dose had no effect on success rate. Age appeared to have operated independent of parity. It had actually affected the success rate in a negative manner. This was evident when comparing women less than 35 years old with older ones. The older women had significantly less success rate. This effect on success rate had not been seen with parity. When women with parity more than 5 were compared with those whose parity was 5 or less, no difference was found. Also when the responders to prostaglandin induction were compared with non-responders it was found that the non-responders had a significantly higher number of women whose age was 35 years old or more.

The present study comprised women of different parities. Thirty two per cent of them were grand multiparous women. The results of this study are comparable to those obtained by Yamani and Rouzi(9) in their study on grand multiparous women with unripe cervices using $1.5 \mathrm{mg}$ dose regimen. Unlike our study, they used amniotomy and syntocinon stimulation to augment labour in $24.8 \%$ of their patients. Ninety per cent of their patients received three applications or less of $1.5 \mathrm{mg}$ PGE2 vaginal tablets. They had a success rate of $88.1 \%$ and one incidence of ruptured uterus. Albar et al.(10), in a study on high parity women from the same region as ours, using a low dose of $1.5 \mathrm{mg}$ prostaglandin $\mathrm{E}_{2}$ tablets but with stepwise increase in the dose, also reported a success rate similar to the success rate in this study. They used syntocinon augmentation in $17.5 \%$ of their patients. However $71 \%$ of their patients required only low doses of 1.5-4.5 mg of prostaglandin. There were no reports of uterine hyperstimulation or rupture in their study. In our study no syntocinon augmentation was used and the total prostaglandin dose received by the patients was less than that of Albar et al (10). In our study $70.8 \%$ of patients from the low dose group who had successful inductions received only one or two doses of $1.5 \mathrm{mg}$ 
prostaglandin. Probably the stepwise increase in the prostaglandin dose is not necessary. In both studies mentioned above no comparison was made between different doses of prostaglandin.

In this study there were two incidences of uterine hyperstimulation and two incidences of ruptured uterus both in the high dose group. The number was too small to give meaningful interpretation. Our small sample size has an inadequate power to assess the impact of induction of labour on these rare outcomes; nevertheless rupture of the uterus is a serious event that calls for caution in the use of prostaglandin in multiparous women. It is interesting that both of them had a posterior uterine rupture. Posterior uterine rupture had been reported with prostaglandin $\mathrm{E}_{2}(4,5)$ and also with misoprostol(11) and syntocinon use(12). Spontaneous posterior rupture of the unscarred uterus without the use of pharmacological agents has also been reported(13). A preliminary report by Yamani and Rouzi(14) about induction of labour in 26 grand multiparous women with one previous caesarean section using $1.5 \mathrm{mg}$ of prostaglandin E2 6 hourly up to 3 doses, depending on uterine and cervical response, and without using oxytocin augmentation, revealed successful vaginal birth in $76.9 \%$ of the patient and no incidence of uterine rupture or any other serious maternal morbidity. However, rupture of the uterus with even small doses of prostaglandin had been reported in other studies $(15,16)$. Absolute safety with prostaglandins can not be claimed whatever formulation, method or dose used. Caution should always be exercised when these agents are used. Using a low dose regimen may help to reduce the incidence of serious complications with an obvious economical advantage. The use of syntocinon augmentation does not seem to be necessary as the results of this study has shown. Avoiding early amniotomy and syntocinon stimulation would render induction of labour simpler, convenient, easier to supervise, with less chances of intrauterine infection and fetal asphyxia and no doubt with less costs. Misoprostol (prostaglandin $\mathrm{El}$ analog) is a cheaper drug with advantages similar to that of prostaglandin E2 vaginal tablets but there still remains a debate about dosage and route of administration(17). Furthermore it is not yet licensed for obstetric use in some countries. In a recent Cochrane review Kelly et al.(18), analysing studies on induction of labour with prostaglandin E2 and F $2 \alpha$ also came to the conclusion that low doses of prostaglandins appear to be as efficacious as high doses and they called for further research towards the cost-analysis of induction of labour with vaginal prostaglandin, with special attention to different methods of administration. Though this study confirms the adequacy of low dose prostaglandin, a limitation of the study is that it is retrospective and the sample size is smaller than desired. Future randomized prospective studies encompassing larger samples are needed to address this important issue.

\section{REFERENCES}

1. Yawn, B.P., Wollan, P., Mckeon, K. and Field, C.S. Temporal changes in rates and reasons for medical induction of term labor. Amer. J. Obst. Gynecol. 2001; 184:611-619.

2. Abouelleil, L.A.A., Nasrat, A.A. and Fayed, H.M., Prostaglandin E2 vaginal pessaries in the grandmultipara with an unripe cervix, a comparison of different parity groups. Int. J. Gynaecol. Obstet. 1993; 40:119-122.

3. Egarter, C.H., Husslein, P.W. and Raybum, W.F. Uterine hyperstimulation after low dose prostaglandin E2 therapy:tocolytic treatment. in 181 cases. Amer. J. Obst. Gynae. 1990; 163:794-796.

4. Maymon R., Haimovich L., Shulman A. et al. Third trimester uterine rupture after prostaglandin E2 use for induction of labour. J. Reprod. Med. 1992; 37:449-452.

5. Hawe, J.A. and Olah, K.S. Posterior uterine rupture in a patient with a lower segment caesarean section scar complicating prostaglandin induction of labour. Hawe. Brit. J. Obstet. Gynaecol. 1997; 104:857-858.

6. Lydon-Rochelle, M., Holt, V.L., Easterling, T.R. and Martin, D.P. Risk of uterine rupture during labor among women with a prior cesarean delivery. N. Engl. J. Med. 2001; 345:3-8.

7. Ravasia, D., Wood, S. and Pollard, J. Uterine rupture during induced trial of labor among women with previous cesarean delivery. Amer. J. Obst. Gynae. 2000; 183:1176-1179.

8. Rimm, A., Hantz, A., Kalbfleich, J., Anderson, A. and Hoffman, R. Basic Biostatistics In Medical epidemiology, in Rimm A (ed): 1980.

9. Yamani, T.Y. and Rouzi, A.A. Induction of labor with vaginal prostaglandin-E2 in grand multiparous women. Int. J. Gynae. Obstet. 1998; 62:255-259.

10. Albar, H., Sobande, A., Hussein, O., Thiga, R. and Mushait, M. The experience with prostaglandin E2 vaginal tablets for induction of labour in grand and great grand multiparae. A two year review in Saudi Arabia. J. Obstet. Gynae. 2000; 20:132-135.

11. Bennett, B.B. Uterine rupture during induction of labor at term with intravaginal misoprostol. Obstet. Gynae. 1997; 89:832-833.

12. Sweeten, K., Graves, W. and Athanassiou, A. Spontaneous rupture of the unscarred uterus. Amer. J. Obstet. Gynae. 1995; 172:1851-1856.

13. Fischer, R.L. Spontaneous rupture of an unscarred uterus in a primigravid woman. Amer. J. Obstet. Gynae. 1996; 175:504-505.

14. Yamani, T.Y. and Rouzi, A.A. Induction of labor with vaginal prostaglandin-E2 in grand multiparous women with one previous cesarean section. Int. J. Gynecol. Obstet. 1998; 65:251-253.

15. Azem, F., Jaffa, A., Lessing, J.B. and Peyser, M.R. Uterine rupture with the use of a lowdose vaginal PGE2 tablet. [see comments]. Acta. Obstet. Gynecol. Scandinavica. 1993; 72:316-317.

16. Voss, D.H., Cumminskyk, K.C., Cook, V.D., Nethers, M.S., Spinnato, J.A. and Gall, S.A. Effects of three concentrations of intracervical prostaglandin gel for cervical ripening.

J. Maternal and Fetal Medi. 1996; 5:186-193.

17. Wing DA: Labor induction with misoprostol. Amer. J. Obstet. Gynae. 1999; 181:339-345.

18. Kelly, A.J., Kavanagh, J. and Thomas, J. Vaginal prostaglandin (PGE2 and PGF2 $\alpha$ ) for induction of labour at term (Cochrane Review). Cochrane Database Syst. Rev. 2: 2001 . 